\section{Histoire Épistémologie Langage}

43-2 | 2021

Linguistique psychologique et sémiotique : le contexte allemand et son influence

\title{
Robert, Jean-Michel. 2020. Leibniz et les universaux
} du langage

Paris : Honoré Champion. 222 p.

\author{
Justin E. H. Smith
}

\section{OpenEdition}

\section{Journals}

Édition électronique

URL : https://journals.openedition.org/hel/1402

DOI : $10.4000 /$ hel. 1402

ISSN : 1638-1580

Éditeur

Société d'histoire et d'épistémologie des sciences du langage

\section{Édition imprimée}

Date de publication : 31 décembre 2021

Pagination : 192-196

ISBN : 9791091587150

ISSN : 0750-8069

Référence électronique

Justin E. H. Smith, «Robert, Jean-Michel. 2020. Leibniz et les universaux du langage », Histoire Épistémologie Langage [En ligne], 43-2 | 2021, mis en ligne le 01 décembre 2021, consulté le 17 décembre 2021. URL : http://journals.openedition.org/hel/1402 ; DOI : https://doi.org/10.4000/hel. 1402

Ce document a été généré automatiquement le 17 décembre 2021.

\section{c) (i)}

HEL is licensed under a Creative Commons Attribution-NonCommercial-NoDerivatives 4.0 International License 


\title{
Robert, Jean-Michel. 2020. Leibniz et les universaux du langage
}

Paris : Honoré Champion. $222 \mathrm{p}$.

\author{
Justin E. H. Smith
}

\section{RÉFÉRENCE}

Robert, Jean-Michel. 2020. Leibniz et les universaux du langage. Paris : Honoré Champion. 222 p. ISBN : 978-2-7453-5392-4.

1 Le rôle de G. W. Leibniz (1646-1716) dans le développement de la linguistique comparée est un sujet relativement peu étudié de nos jours. Les études portant sur sa philosophie générale, publiées depuis le siècle dernier, ne mentionnent tout au plus que dans quelques notes de bas de page son intérêt pour la recherche systématique sur la diversité et sur la parenté des langues. Les grands travaux $\mathrm{du} \mathrm{xx}^{\mathrm{e}}$ siècle portant sur cette question, notamment Leibniz als Sprachforscher de Sigrid von der Schulenburg, écrit dans les années 1930 et publié seulement en 1973, sont généralement respectés par les leibniziens, mais leur place dans la recherche sur Leibniz est considérée comme étant de moindre importance que ceux portant sur la « philosophia prima » de Leibniz.

Pourtant, dans la première réception du philosophe, les chercheurs étaient beaucoup plus prompts à reconnaître et à valoriser cette dimension de son œuvre. La première publication posthume de textes choisis, faite par Joachim Friedrich Feller sous le titre Otium Hanoveranum sive Miscellanea en 1718, rassemble en un volume de nombreux courts écrits portant sur des questions historico-linguistiques. De même, la grande édition genevoise des Opera omnia, faite par Louis Dutens en 1768, dont un grand pourcentage des « opera » (qui sont, malgré le titre, loin d'être « omnia ») sont consacrés à des recherches sur les origines des nations européennes par le moyen de comparaison et de classification de leurs langues, un genre d'ethnolinguistique généalogique qui inclut des textes comme le De originibus nationum Transylvaniae et la Dissertatio de origine Germanorum. En jugeant des priorités éditoriales de Dutens, un lecteur pourrait 
facilement en tirer la conclusion que la contribution de Leibniz a été avant tout dans les champs de la linguistique et de l'ethnologie. Ce n'est qu'au XIX ${ }^{e}$ siècle que cette image de Leibniz commence à s'affadir (même si Max Müller et d'autres linguistes de l'époque la conservent) avec la publication de ses Philosophische Schriften par C. I. Gerhardt entre 1875 et 1890.

3 En somme, il est loin d'être évident que l'image qui règne tout au long du $\mathrm{xx}^{\mathrm{e}}$ siècle d'un Leibniz avant tout logicien et métaphysicien soit plus fidèle au penseur lui-même que celle qui la précède, et il est important que la recherche leibnizienne d'aujourd'hui renoue avec le Leibniz de Feller et de Dutens pour mieux comprendre toute la gamme de ses intérêts. D'autant plus que, comme nous le montre le nouveau livre de JeanMichel Robert, les deux Leibniz sont en réalité une seule et même personne: les recherches qu'il mène sur la diversité des langues sont soutenues et inspirées par ses convictions métaphysiques les plus fondamentales, comme l'idée selon laquelle le monde consiste en "diversité compensée par l'identité », autrement dit qu'il existe une unité derrière toute la différence apparente. Pour les communautés linguistiques comme pour les monades, étudier l'une peut nous aider à comprendre l'autre ; le projet philosophique de Leibniz est en soi une " unité diverse ».

4 Malgré ses nombreux atouts sur lesquels nous reviendrons, l'ouvrage de J.-M. Robert ne s'intéresse pas de prime abord aux idées de Leibniz dans leur contexte historique ni dans leur développement. Même si son choix des idées abordées peut être dans une grande mesure justifié par les objectifs internes du livre, le lecteur aurait tout de même voulu en savoir plus sur les connaissances concrètes et les méthodes pratiques que Leibniz adoptait en matière de recherche linguistique. L'auteur mentionne à peine la méthodologie de Leibniz en ethnolinguistique comparée, malgré l'insistance du philosophe de Hanovre sur la nécessité du travail de terrain et de la récolte d'échantillons de langues, travail analogue à la récolte des espèces de fleurs qu'un botaniste presse entre les pages d'un livre. Il s'agit ici d'une méthode que Leibniz a apprise grâce aux missionnaires jésuites qui collectionnaient des échantillons du Pater Noster en version bilingue avec le texte interlinéaire en russe, latin, néerlandais ou une autre langue occidentale. Leibniz comprend que toute la valeur de cette approche réside dans la possibilité d'un échange réciproque, dans lequel les peuples rencontrés reçoivent la vérité de la foi chrétienne, tandis que les chercheurs européens reçoivent dans chaque version de la prière de nouvelles données pour reconstruire le système de parenté entre les langues humaines.

Il est hors de doute que ce sont les relations avec les conseillers de Pierre le Grand et les voyageurs dans l'empire russe, à partir des années 1690, qui donnèrent à Leibniz l'opportunité d'appliquer cette méthode. Pour lui, la Russie représente un grand laboratoire pour l'étude de la linguistique comparée et pour la détermination des liens de parenté possibles entre les langues dites "sarmatiques" (slaves), germaniques, finno-ougriennes et turciques. Leibniz commence à briguer un poste à la cour russe autour de 1698 et réalise son ambition en 1712 en devenant conseiller privé du tsar, dans le but exprès d'aider à faire avancer les savoirs scientifiques dans ce pays et à organiser la fondation de l'Académie de Saint-Pétersbourg (qui sera finalement fondée en 1725, neuf ans après sa mort). Les deux grandes expéditions à travers l'empire, entre 1725 et 1741, appelées souvent les "expéditions du Kamtchatka», peuvent être comprises comme la réalisation posthume, à une grande échelle, de sa vision de la recherche en linguistique comparée. Il est donc à regretter que J.-M. Robert consacre 
relativement peu de place aux liens de Leibniz avec la Russie. Le peu de littérature qu'il cite sur ce sujet est très datée (surtout le livre de Liselotte Richter de 1946), et il ne semble pas avoir pris en compte les nouvelles publications à ce sujet, comme le récent livre de Han F. Vermeulen, Before Boas: The Genesis of Ethnography and Ethnology in the German Enlightenment. J.-M. Robert ne mentionne pas non plus les correspondances importantes que Leibniz a entretenue avec J. G. Sparwenfeld, l'auteur suédois du grand Lexicon Slavonicum, ni avec Nicolaes Witsen, le voyageur néerlandais et fournisseur pour Leibniz de nombreuses versions du Pater Noster.

6 Cette dimension du travail linguistique de Leibniz pourrait sembler éloignée des enjeux de la perspective de J.-M. Robert, mais, comme c'est souvent le cas chez Leibniz, la méthode que celui-ci emploie ou propose pour appliquer ses hypothèses théoriques nous révèle des dimensions de la théorie elle-même qui restent cachées si nous ne faisons pas suffisamment attention à ses pratiques concrètes. Dans le cas dont il est question, il est clair que c'est dans l'étude de la diversité des langues sur le terrain (même si Leibniz lui-même ne quitte pas son fauteuil) que le philosophe s'éloigne graduellement de son postulat original d'une langue parfaite donnée par Dieu à l'humanité. J.-M. Robert écrit parfois comme si Leibniz maintenait ce postulat d'une manière figée et inchangeante tout au long de sa carrière. Or, en réalité, comme toute idée que Leibniz entretient, la question de la langue originelle évolue dans sa pensée et elle semble être au cours du temps dans une proportion inverse avec le projet de linguistique comparée sur le terrain : plus Leibniz est occupé par le projet de ramasser des échantillons du Pater Noster, moins il est préoccupé par la question de la langue originelle que ce même Pater est censé nous avoir léguée. Si J.-M. Robert a donc raison d'écrire que Leibniz " postule l'existence d'une langue originelle parfaite », cela dépend entièrement de la période de la longue carrière du philosophe en question.

7 Le livre de J.-M. Robert est divisé en trois parties. Si une bonne part de la première, esquissant le parcours de Leibniz, nous a semblé hors de propos étant donné la quantité de sources déjà existantes pour le lecteur désireux de se familiariser avec la biographie du philosophe, la deuxième partie du livre, qui porte sur la théorie leibnizienne de la langue universelle, et la troisième, consacrée à sa réflexion sur les universaux linguistiques, nous ont paru beaucoup mieux conçues et réalisées.

Dans la première partie, après l'esquisse du parcours du philosophe, l'auteur résume ses recherches et réflexions autour des langues et du langage. Il organise cette discussion en trois sections sous les rubriques de "Lexis ", "Logos » et "Logique ». Comme J.-M. Robert l'explique, pour la plupart des contemporains de Leibniz, le candidat le plus évident pour le rôle de langue originelle, c'est l'hébreu. Cette langue biblique est souvent confondue ou mélangée avec l'idée d'une "langue adamique", c'est-à-dire avec la langue supposément parlée par Adam et Ève avant le péché originel et censée exprimer directement et de façon non arbitraire les essences des êtres qu'elle nomme. Or, pour Leibniz, il est évident que toutes les langues naturelles sont le produit d'une histoire humaine particulière, et même s'il y en a qui sont plus " primitives » que les autres et qui représentent pour ainsi dire des fossiles linguistiques vivants, cela ne signifie pas que celles-ci doivent être considérées comme plus proches de la langue divine ni des essences des choses. Comme l'explique J.-M. Robert: "[L]a grande originalité de Leibniz est d'avoir su assigner à l'hébreu une place à l'intérieur d'un système, plutôt que de l'avoir considéré comme clef du voûte du système » (p. 51). Cette conviction va de pair avec la recherche que Leibniz mène sur la characteristica 
universalis et sur une grammaire rationnelle: une fois que nous constatons que ni l'hébreu ni aucune autre langue humaine n'occupe une place spéciale parmi les autres, nous pouvons arrêter de regarder « en arrière " pour le projet de réaliser un système de communication idéal.

Dans la deuxième partie du livre, J.-M. Robert s'intéresse au projet de langue universelle de Leibniz, en commençant par ses précurseurs immédiats, tels René Descartes, George Dalgarno et John Wilkins. Il passe ensuite à un examen très fin du projet de caractéristique rationnelle chez Leibniz, ainsi qu'à ses recherches sur les systèmes d'écriture idéographique. Ici encore, de telles réflexions sont inséparables, pour lui comme pour ses contemporains, du contexte ethnographique et de l'histoire du contact interculturel à cette époque, avant tout avec la Chine, mais aussi avec le Mexique, l'aire culturelle andine, etc. Or l'auteur ne cite presque aucune source de la littérature secondaire consacrée à l'influence de la culture textuelle chinoise sur la pensée de Leibniz, pas même les grands travaux de David Mungello.

10 Ensuite, J.-M. Robert se tourne vers la question d'une grammaire rationnelle chez Leibniz, conçue comme une forme de logique sémantico-syntaxique. Le projet leibnizien de construire une telle grammaire se décline en plusieurs parties, dont la réduction des difficultés du latin et leur remplacement par un système uniforme de conjugaison avec un seul genre et un seul nombre, la transformation de tous les verbes en une forme être + participe, etc. Pour les fonctions syntaxiques, à leur tour, Leibniz s'inspire largement de la logique d'Aristote, courant ainsi (Yvon Belaval l'avait bien noté) le même risque que son prédécesseur de confondre les actes intellectuels et les actes grammaticaux.

11 J.-M. Robert explique bien les raisons pour lesquelles Leibniz ne croit pas qu'une langue universelle, avec une grammaire rationnelle parfaite, puisse jamais être réalisée. Exprimer des vérités en une telle langue serait du même coup atteindre la rationalité divine. Mais son projet de construire une grammaire relativement plus rationnelle que celles des langues naturelles héritées sert néanmoins comme modèle et inspiration dans les programmes de formation de langues artificielles jusqu'à nos jours.

Dans la troisième et dernière partie, J.-M. Robert essaie de mesurer les contributions de Leibniz par le prisme de la linguistique (théorique et appliquée) contemporaine. Il commence par une discussion de la sémiotique, basée notamment sur les travaux de Saussure, Benjamin et Kristeva, puis il présente la littérature secondaire récente sur les langues et les grammaires universelles, en essayant de mettre Leibniz en dialogue avec, entre autres, Noam Chomsky. Cette discussion s'avère particulièrement riche.

13 Pour Leibniz, une grammaire universelle se construit, d'une part, à partir de la "grammaire-noyau » ou de la réduction d'une langue naturelle particulière, et, d'autre part, avec les transformations qui donnent à ce système réduit une syntaxe logique. La réduction pour lui fait partie du projet plus large de construire une grammaire philosophique en éliminant toutes les imperfections qui compromettent la clarté d'une grammaire naturelle quelconque. Pour Chomsky, par contre, une grammaire universelle n'est rien d'autre qu'une capacité innée pour la programmation linguistique abstraite qui elle-même produit les grammaires-noyaux, à savoir la "structure profonde » du langage. En dépit des différences réelles, d'après l'auteur, Chomsky reste l'héritier de l'approche leibnizienne dans la mesure où il conserve la distinction fondamentale entre lexis et logique, c'est-à-dire entre dénomination et relation. 
Enfin ce que Leibniz essaie de faire dans son projet linguistique, c'est d'envisager une langue universelle qui permettra un "retour à une dénomination première " et une réduction et transformation d'une langue naturelle "selon l'ordre naturel des idées ». J.-M. Robert conclut donc que « les universaux du langage [...] se confondent [...] avec les universaux de la logique humaine, elle-même reflet pour Leibniz d'une logique supérieure » (p. 204). Nous sommes ici loin de la linguistique chomskyenne, mais en mettant les deux penseurs en comparaison, J.-M. Robert nous aide à mieux apprécier les enjeux philosophiques dans la longue durée lorsque Chomsky se qualifie lui-même de « rationaliste ». Autrement dit, J.-M. Robert nous montre combien l'histoire de la linguistique peut être pertinente pour une compréhension approfondie du rationalisme philosophique.

\section{BIBLIOGRAPHIE}

Leibniz, G. W. 1718. Otium Hanoveranum sive Miscellanea, Ex ore \& schedis Illustris Viri, piae memoriae, Godofr. Guilelmi Leibnitii. Éd. par Joachim Friedrich Feller. Leipzig : Imprensis Joann. Christiani Martini.

Leibniz, G. W. 1768. Gothofredi Guillelmi Leibnitii Opera Omnia. Nunc primum collecta. Éd. par Louis Dutens. 6 vol. Genève : Fratres De Tournes.

Leibniz, G. W. 1875-90. Die philosophischen Schriften von G. W. Leibniz. Éd. par C. I. Gerhardt. 7 vol. Berlin : Weidmann'sche Buchhandlung.

Richter, Liselotte. 1946. Leibniz und sein Russlandbild. Berlin : Akademie der Wissenschaften zu Berlin.

Schulenburg, Sigrid von der. 1973. Leibniz als Sprachforscher. Francfort-sur-le-Main :

V. Klostermann.

Vermeulen, Han F. 2015. Before Boas: The Genesis of Ethnography and Ethnology in the German Enlightenment. Lincoln \& Londres : University of Nebraska Press.

\section{AUTEURS}

\section{JUSTIN E. H. SMITH}

Laboratoire Sphere, département Histoire et philosophie des sciences de l’Université de Paris 